\title{
ANALYTICAL INVESTIGATION FOR MODIFIED RIEMANN-LIOUVILLE FRACTIONAL EQUAL-WIDTH EQUATION TYPES BASED ON $\left(G^{\prime} / G\right)$ - EXPANSION TECHNIQUE
}

\author{
BAHAR KARAMAN
}

Received 07 January, 2020

\begin{abstract}
The present investigation studies exact solutions of modified Riemann-Liouville fractional Equal-Width (MRLFEW) equation types with the help of the $\left(G^{\prime} / G\right)$ - expansion method. Firstly, the MRLFEW equation is converted into an ordinary differential equation via fractional complex transform. Then, the proposed method has applied this equation to construct the exact solutions.
\end{abstract}

2010 Mathematics Subject Classification: 34K37; 34A08; 26A33

Keywords: $\left(G^{\prime} / G\right)$-expansion technique, fractional EW equations, modified Riemann-Liouville fractional (MRLF)

\section{INTRODUCTION}

In recent years, real-world phenomena can be modeled successfully by the partial differential equations with fractional derivatives (FPDEs). These equations have significant applications in various areas such as fluid mechanics, viscoelastic materials, finance, control theory, fractional dynamics, biology, physics, applied mathematics and engineering $[1,3,4,6,8,10,13,18]$. Many studies have been introduced by many researchers $[5,7,15,17]$. To seek exact solutions of the FPDEs, many well-known methods have been proposed such as the exp-function method [16], modified simple method [21], the fractional sub-equation method [2,23], $\left(G^{\prime} / G\right)$-expansion method [20,22].

In this manuscript, we are mainly concerned to explore traveling wave solutions of MRLFEW equation types. So far, various analytical methods have been suggested for the equation types by the references therein [11,14,19]. To our knowledge, $\left(G^{\prime} / G\right)$-expansion technique is not used to generate the traveling wave solutions of MRLFEW equation types. Adding to this, the advantages of this technique are ease of implementation and obtaining new solutions. That's why we implement the technique to construct exact solutions to the mentioned equations. The layout of this work is as follows: In Section 1, a brief of the MRLF derivative is given. The next Section 
2 gives the description of the $\left(G^{\prime} / G\right)$-expansion methodology. In Section 3, we construct the implementation of the suggested method for the MRLFEW equation types. The results are also illustrated in this section. Finally, we end with a short conclusion in Section 4.

\subsection{A Brief of the MRLF derivative}

Jumarie is expressed $\alpha-$ th order fractional derivative in sense of MRL of a given function $y=v(t)$ as

$$
D_{t}^{\alpha} v(t)= \begin{cases}\frac{1}{\Gamma(1-\alpha)} \frac{d}{d t} \int_{0}^{t}(t-\xi)^{-\alpha}(v(\xi)-v(0)) d \xi, & 0<\alpha<1, \\ \left(v^{(m)}(t)\right)^{\alpha-m}, & m \leq \alpha<m+1, \quad m \geq 1\end{cases}
$$

This definition has some prominent properties. They are given [12] following as

$$
\begin{array}{r}
D_{t}^{\alpha} t^{p}=\frac{\Gamma(p+1)}{\Gamma(p+1-\alpha)} t^{\alpha-p}, \quad p>0 \\
D_{t}^{\alpha}(f(t) g(t))=f(t) D_{t}^{\alpha} g(t)+g(t) D_{t}^{\alpha} f(t) .
\end{array}
$$

\section{THE $\left(G^{\prime} / G\right)$ - EXPANSION METHODOLOGY}

We outline the basic idea of the $\left(G^{\prime} / G\right)$ - expansion technique. Firstly, a general nonlinear fractional differential equation with two independent variables $x$ and $t$ is regarded as

$$
R\left(v, D_{t}^{\alpha} v, D_{x}^{\beta} v, D_{t}^{\alpha} D_{t}^{\alpha} v, D_{t}^{\alpha} D_{x}^{\beta} v, D_{x}^{\beta} D_{x}^{\beta} v, \cdots\right)=0, \quad 0<\alpha, \beta<1,
$$

in here $v$ is an unknown function, $R$ is a polynomial of $v$ and its various partial derivatives containing the highest order derivatives and nonlinear terms.

There are main steps to implement the proposed method. These are as follows.

Stage 1: A nonlinear fractional complex transformation proposed by $\mathrm{Li}$ and $\mathrm{He}$ [9] is used to reduce fractional differential equations into ordinary differential equations (ODEs). This transformation is described as

$$
v(x, t)=V(\xi), \quad \xi=\frac{c_{1} x^{\beta}}{\Gamma(1+\beta)}-\frac{c_{2} t^{\alpha}}{\Gamma(1+\alpha)}
$$

where $c_{1}$ and $c_{2}$ are arbitrary constants. We would also like to note that the chain rule can be computed as

$$
\begin{aligned}
& D^{\alpha}{ }_{t} v=\sigma_{1} \frac{d V}{d \xi} D_{t}^{\alpha \xi} \\
& D^{\beta}{ }_{x} v=\sigma_{2} \frac{d V}{d \xi} D_{x}^{\beta \xi}
\end{aligned}
$$


where $\sigma_{1}, \sigma_{2}$ are fractional indexes [9]. Eqn. (2.1) can be rewritten by using Eqns. (2.2) and the chain rule (2.3)-(2.4) as the following ODE:

$$
F\left(V, V^{\prime}, V^{\prime \prime}, V^{\prime \prime \prime}, \cdots\right)=0
$$

in here the prime denotes the derivation with respect to $\xi$.

Stage 2: Assuming the exact solution of Eqn. (2.5) can be represented by a polynomial in $\left(G^{\prime} / G\right)$ as the following form:

$$
v(\xi)=\sum_{j=0}^{p} b_{j}\left(\frac{G^{\prime}}{G}\right)^{j}, \quad b_{p} \neq 0
$$

where $b_{0}, b_{1}, \cdots, b_{p}$ are constants. Also, $G(\xi)$ satisfies the second-order linear ODE which is defined by

$$
G^{\prime \prime}(\xi)+\lambda_{1} G^{\prime}(\xi)+\lambda_{2} G(\xi)=0
$$

with $\lambda_{1,2}$ being arbitrary constants. Balancing between the highest order derivatives and the nonlinear term rising in Eqn. (2.5) is used to determine the balancing number $p$.

Stage 3: In this step, we first substitute Eqn. (2.6) into Eqn. (2.5) and use Eqn. (2.7). Then, we gather up all the coefficients with the same power of $\left(G^{\prime} / G\right)$. Equalizing each term of the obtaining polynomial to zero yields a set of algebraic equations for $b_{0}, b_{1}, \cdots, b_{p}, \lambda_{1}, \lambda_{2}, c_{1}$, and $c_{2}$.

Stage 4: We construct the constants $b_{0}, b_{1}, \cdots, b_{p}, \lambda_{1}, \lambda_{2}, c_{1}$ and $c_{2}$ by solving the obtained system. Substituting these parameters along with the general solution of Eqn. (2.7) into Eqn. (2.6), a variety exact solutions of Eqn. (2.1) is obtained.

\section{IMPLEMENTATION OF THE SUGGESTED METHOD FOR MRLFEW EQUATION TYPES}

The main aim of this part is to solve MRLFEW equation types based on the abovementioned methodology. We consider the equation types that is defined as follows

$$
D_{t}^{\alpha} v(x, t)+\varepsilon D_{x}^{\alpha} v^{2}(x, t)-\delta D_{x x t}^{3 \alpha} v(x, t)=0
$$

and

$$
D_{t}^{\alpha} v(x, t)+\varepsilon D_{x}^{\alpha} v^{3}(x, t)-\delta D_{x x t}^{3 \alpha} v(x, t)=0
$$

where $\varepsilon$ and $\delta$ are real parameters and $\alpha$ is the order of MRLF derivative. The first equation is known as fractional EW equation. The second is called modified fractional EW equation. 


\subsection{Fractional EW equation}

By substituting the transformation (2.2) into Eqn. (3.1), the following ODE can be found

$$
-c V^{\prime}+\varepsilon k\left(V^{2}\right)^{\prime}+\delta c k^{2} V^{\prime \prime \prime}=0
$$

where $c=-\sigma_{1} c_{2}$ and $k=\sigma_{2} c_{1}$. Let's integrate Eqn. (3.3) once and setting the integration constant to zero yield

$$
-c V+\varepsilon k V^{2}+\delta c k^{2} V^{\prime \prime}=0
$$

Eqn. (3.4) gives the balancing number,

$$
m+2=2 m,
$$

so

$$
m=2 \text {. }
$$

We assume that the solution of Eqn. (3.4) can be described by a polynomial in $\left(G^{\prime} / G\right)$ as follows:

$$
V(\xi)=b_{0}+b_{1}\left(\frac{G^{\prime}}{G}\right)+b_{2}\left(\frac{G^{\prime}}{G}\right)^{2}, \quad b_{2} \neq 0
$$

Using Eqn. (2.7) and Eqn. (3.5), we have

$$
\begin{aligned}
V^{2}(\xi) & =b_{0}^{2}+b_{1}^{2}\left(\frac{G^{\prime}}{G}\right)^{2}+b_{2}^{2}\left(\frac{G^{\prime}}{G}\right)^{4}+2 b_{0} b_{1}\left(\frac{G^{\prime}}{G}\right) \\
& +2 b_{0} b_{2}\left(\frac{G^{\prime}}{G}\right)^{2}+2 b_{1} b_{2}\left(\frac{G^{\prime}}{G}\right)^{3}, \\
V^{\prime \prime}(\xi) & =\left(b_{1} \lambda_{1}+2 b_{2} \lambda_{2}\right)\left[\left(\frac{G^{\prime}}{G}\right)^{2}+\lambda_{1}\left(\frac{G^{\prime}}{G}\right)+\lambda_{2}\right] \\
& +2\left(b_{1}+2 b_{2} \lambda_{1}\right)\left[\left(\frac{G^{\prime}}{G}\right)^{3}+\lambda_{1}\left(\frac{G^{\prime}}{G}\right)^{2}+\lambda_{2}\left(\frac{G^{\prime}}{G}\right)\right] \\
& +6 b_{2}\left[\left(\frac{G^{\prime}}{G}\right)^{4}+\lambda_{1}\left(\frac{G^{\prime}}{G}\right)^{3}+\lambda_{2}\left(\frac{G^{\prime}}{G}\right)^{2}\right] .
\end{aligned}
$$

Let's substitute Eqns. (3.5)-(3.6) into Eqn. (3.4). Then collecting the coefficients of $\left(G^{\prime} / G\right)^{j},(j=0,1,2,3,4)$ and setting them to be zero, the system is found as form:

$$
\begin{array}{r}
-c b_{0}+e k b_{0}^{2}+b_{1} \lambda_{1} \lambda_{2} \delta c k^{2}+2 b_{2} \lambda_{2}^{2} \delta c k^{2}+\chi_{0}=0, \\
-c b_{1}+2 \varepsilon k b_{0} b_{1}+b_{1} \lambda_{1}^{2} \delta c k^{2}+2 b_{2} \lambda_{1} \lambda_{2} \delta c k^{2} \\
+2 b_{1} \lambda_{2} \delta c k^{2}+4 b_{2} \lambda_{1} \lambda_{2} \delta c k^{2}=0, \\
-c b_{2}+\varepsilon k b_{1}^{2}+2 \varepsilon k b_{0} b_{2}+b_{1} \lambda_{1} \delta c k^{2}+2 b_{2} \lambda_{2} \delta c k^{2} \\
+2 b_{1} \lambda_{1} \delta c k^{2}+4 b_{2} \lambda_{1}^{2} \delta c k^{2}+6 b_{2} \lambda_{2} \delta c k^{2}=0,
\end{array}
$$




$$
\begin{aligned}
2 \varepsilon k b_{1} b_{2}+2 b_{1} \delta c k^{2}+4 b_{2} \lambda_{1} \delta c k^{2}+6 b_{2} \lambda_{1} \delta c k^{2} & =0, \\
\varepsilon k b_{2}^{2}+6 \delta c k^{2} b_{2} & =0 .
\end{aligned}
$$

This system is solved by using Maple, we obtain

$$
\begin{aligned}
& b_{0}=-\frac{c\left(k^{2} \lambda_{1}^{2} \delta+8 k^{2} \lambda_{2} \delta-1\right)}{2 k \varepsilon}, \quad b_{1}=-\frac{6 c k \delta \lambda_{1}}{\varepsilon}, \quad b_{2}=-\frac{6 c k \delta}{\varepsilon} \\
& \delta=\delta, \quad \varepsilon=\varepsilon, \quad c=c, \quad k=k .
\end{aligned}
$$

where $\lambda_{1}$ and $\lambda_{2}$ are arbitrary constants. Eqn. (3.5) can be rewritten by using Eqn. (3.13) as follows:

$$
V(\xi)=-\frac{c\left(k^{2} \lambda_{1}^{2} \delta+8 k^{2} \lambda_{2} \delta-1\right)}{2 k \varepsilon}-\frac{6 c k \delta \lambda_{1}}{\varepsilon}\left(\frac{G^{\prime}}{G}\right)-\frac{6 c k \delta}{\varepsilon}\left(\frac{G^{\prime}}{G}\right)^{2}
$$

The general solution of Eqn. (2.7) is substituted into Eqn. (3.14), we get three types of travelling wave solutions of the Eqn. (3.1) as follows:

When $\lambda_{1}^{2}-4 \lambda_{2}>0$

$$
V_{1}(\xi)=-\frac{c\left(k^{2} \lambda_{1}^{2} \delta+8 k^{2} \lambda_{2} \delta-1\right)}{2 k \varepsilon}-\frac{6 c k \delta \lambda_{1}}{\varepsilon}\left(\frac{G^{\prime}}{G}\right)-\frac{6 c k \delta}{\varepsilon}\left(\frac{G^{\prime}}{G}\right)^{2},
$$

where

$$
\left(\frac{G^{\prime}}{G}\right)=-\frac{\lambda_{1}}{2}+\frac{\sqrt{\lambda_{1}^{2}-4 \lambda_{2}}}{2}\left(\frac{C_{1} \cosh \left(\frac{\sqrt{\lambda_{1}^{2}-4 \lambda_{2}}}{2}\right) \xi+C_{2} \sinh \left(\frac{\sqrt{\lambda_{1}^{2}-4 \lambda_{2}}}{2}\right) \xi}{C_{1} \sinh \left(\frac{\sqrt{\lambda_{1}^{2}-4 \lambda_{2}}}{2}\right) \xi+C_{2} \cosh \left(\frac{\sqrt{\lambda_{1}^{2}-4 \lambda_{2}}}{2}\right) \xi}\right)
$$

When $\lambda_{1}^{2}-4 \lambda_{2}<0$,

$$
V_{2}(\xi)=-\frac{c\left(k^{2} \lambda_{1}^{2} \delta+8 k^{2} \lambda_{2} \delta-1\right)}{2 k \varepsilon}-\frac{6 c k \delta \lambda_{1}}{\varepsilon}\left(\frac{G^{\prime}}{G}\right)-\frac{6 c k \delta}{\varepsilon}\left(\frac{G^{\prime}}{G}\right)^{2},
$$

where

$$
\left(\frac{G^{\prime}}{G}\right)=-\frac{\lambda_{1}}{2}+\frac{\sqrt{4 \lambda_{2}-\lambda_{1}^{2}}}{2}\left(\frac{C_{1} \cos \left(\frac{\sqrt{4 \lambda_{2}-\lambda_{1}^{2}}}{2}\right) \xi-C_{2} \sin \left(\frac{\sqrt{4 \lambda_{2}-\lambda_{1}^{2}}}{2}\right) \xi}{C_{1} \sin \left(\frac{\sqrt{4 \lambda_{2}-\lambda_{1}^{2}}}{2}\right) \xi-C_{2} \cos \left(\frac{\sqrt{4 \lambda_{2}-\lambda_{1}^{2}}}{2}\right) \xi}\right)
$$

When $\lambda_{1}^{2}-4 \lambda_{2}=0$,

$$
V_{3}(\xi)=-\frac{c\left(k^{2} \lambda_{1}^{2} \delta+8 k^{2} \lambda_{2} \delta-1\right)}{2 k \varepsilon}-\frac{6 c k \delta \lambda_{1}}{\varepsilon}\left(\frac{G^{\prime}}{G}\right)-\frac{6 c k \delta}{\varepsilon}\left(\frac{G^{\prime}}{G}\right)^{2}
$$

where

$$
\left(\frac{G^{\prime}}{G}\right)=-\frac{\lambda_{1}}{2}+\frac{C_{2}}{C_{1}+C_{2} \xi} .
$$




\subsection{Modified fractional EW equation}

By substituting the transformation (2.2) into Eqn. (3.2), the following ODE can be found

$$
-c V^{\prime}+\varepsilon k\left(V^{3}\right)^{\prime}+\delta c k^{2} V^{\prime \prime \prime}=0
$$

where $c=\sigma_{1} c_{2}$ and $k=\sigma_{2} c_{1}$. Let's integrate Eqn. (3.18) once, we get

$$
-c V+\varepsilon k V^{3}+\delta c k^{2} V^{\prime \prime}+\chi_{1}=0
$$

where $\chi_{1}$ is integration constant. Eqn. (3.19) gives the balancing number,

$$
m+2=3 m,
$$

so

$$
m=1 \text {. }
$$

We assume that the solution of Eqn. (3.19) can be described by a polynomial in $\left(G^{\prime} / G\right)$ as follows:

$$
V(\xi)=b_{0}+b_{1}\left(\frac{G^{\prime}}{G}\right), \quad b_{1} \neq 0
$$

Using Eqn. (2.7) and Eqn. (3.20), we have

$$
\begin{aligned}
V^{3}(\xi) & = & & b_{0}^{3}+3 b_{0}^{2} b_{1}\left(\frac{G^{\prime}}{G}\right)+3 b_{0} b_{1}^{2}\left(\frac{G^{\prime}}{G}\right)^{2}+b_{1}^{3}\left(\frac{G^{\prime}}{G}\right)^{3} \\
V^{\prime \prime}(\xi) & = & & 2 b_{1}\left(\frac{G^{\prime}}{G}\right)^{3}+3 b_{1} \lambda_{1}\left(\frac{G^{\prime}}{G}\right)^{2} \\
+ & & & \left(b_{1} \lambda_{1}^{2}+2 b_{1} \lambda_{2}\right)\left(\frac{G^{\prime}}{G}\right)+b_{1} \lambda_{1} \lambda_{2}
\end{aligned}
$$

Let's substitute Eqns. (3.20)-(3.21) into Eqn. (3.19). Then collecting the coefficients of $\left(G^{\prime} / G\right)^{j},(j=0,1,2,3)$ and setting them to be zero, the algebraic equation system is found as form:

$$
\begin{aligned}
-c b_{0}+\varepsilon k b_{0}^{3}+\delta c k^{2} b_{1} \lambda_{1} \lambda_{2}+\chi_{1} & =0 \\
-c b_{1}+3 \varepsilon k b_{0}^{2} b_{1}+2 \delta c k^{2} b_{1} \lambda_{2}+\delta c k^{2} b_{1} \lambda_{1}^{2} & =0 \\
3 \varepsilon k b_{0} b_{1}^{2}+3 \delta c k^{2} b_{1} \lambda_{1} & =0 \\
\varepsilon k b_{1}^{3}+2 b_{1} \delta c k^{2} & =0
\end{aligned}
$$

This system is solved by using Maple, we obtain

$$
\begin{array}{r}
b_{0}= \pm \frac{\sqrt{2 \varepsilon c k \delta} \lambda_{1} i}{2 \varepsilon}, \quad b_{1}= \pm \frac{\sqrt{2 \varepsilon c k \delta} i}{2 \varepsilon}, \\
\chi_{1}=0, \quad k= \pm \frac{2}{\sqrt{-2 \lambda_{1}^{2} \delta+8 \lambda_{2} \delta}}, \\
\delta=\delta, \quad \varepsilon=\varepsilon, \quad c=c
\end{array}
$$


where $\lambda_{1}$ and $\lambda_{2}$ are arbitrary constants. Eqn. (3.20) can be rewritten by using Eqn. (3.26) as follows:

$$
V(\xi)= \pm \frac{\sqrt{2 \varepsilon c k \delta} \lambda_{1} i}{2 \varepsilon} \pm \frac{\sqrt{2 \varepsilon c k \delta} i}{2 \varepsilon}\left(\frac{G^{\prime}}{G}\right)
$$

The general solution of Eqn. (2.7) is substituted into Eqn. (3.14), we get three types of travelling wave solutions of the Eqn. (3.1) as follows:

When $\lambda_{1}^{2}-4 \lambda_{2}>0$,

$$
V_{1}(\xi)= \pm \frac{\sqrt{2 \varepsilon c k \delta} \lambda_{1} i}{2 \varepsilon} \pm \frac{\sqrt{2 \varepsilon c k \delta} i}{2 \varepsilon}\left(\frac{G^{\prime}}{G}\right)
$$

where

$$
\left(\frac{G^{\prime}}{G}\right)=-\frac{\lambda_{1}}{2}+\frac{\sqrt{\lambda_{1}^{2}-4 \lambda_{2}}}{2}\left(\frac{C_{1} \cosh \left(\frac{\sqrt{\lambda_{1}^{2}-4 \lambda_{2}}}{2}\right) \xi+C_{2} \sinh \left(\frac{\sqrt{\lambda_{1}^{2}-4 \lambda_{2}}}{2}\right) \xi}{C_{1} \sinh \left(\frac{\sqrt{\lambda_{1}^{2}-4 \lambda_{2}}}{2}\right) \xi+C_{2} \cosh \left(\frac{\sqrt{\lambda_{1}^{2}-4 \lambda_{2}}}{2}\right) \xi}\right)
$$

When $\lambda_{1}^{2}-4 \lambda_{2}<0$,

$$
V_{2}(\xi)= \pm \frac{\sqrt{2 \varepsilon c k \delta} \lambda_{1} i}{2 \varepsilon} \pm \frac{\sqrt{2 \varepsilon c k \delta} i}{2 \varepsilon}\left(\frac{G^{\prime}}{G}\right)
$$

where

$$
\left(\frac{G^{\prime}}{G}\right)=-\frac{\lambda_{1}}{2}+\frac{\sqrt{4 \lambda_{2}-\lambda_{1}^{2}}}{2}\left(\frac{C_{1} \cos \left(\frac{\sqrt{4 \lambda_{2}-\lambda_{1}^{2}}}{2}\right) \xi-C_{2} \sin \left(\frac{\sqrt{4 \lambda_{2}-\lambda_{1}^{2}}}{2}\right) \xi}{C_{1} \sin \left(\frac{\sqrt{4 \lambda_{2}-\lambda_{1}^{2}}}{2}\right) \xi-C_{2} \cos \left(\frac{\sqrt{4 \lambda_{2}-\lambda_{1}^{2}}}{2}\right) \xi}\right)
$$

When $\lambda_{1}^{2}-4 \lambda_{2}=0$,

$$
V_{3}(\xi)= \pm \frac{\sqrt{2 \varepsilon c k \delta} \lambda_{1} i}{2 \varepsilon} \pm \frac{\sqrt{2 \varepsilon c k \delta} i}{2 \varepsilon}\left(\frac{G^{\prime}}{G}\right)
$$

where

$$
\left(\frac{G^{\prime}}{G}\right)=-\frac{\lambda_{1}}{2}+\frac{C_{2}}{C_{1}+C_{2} \xi} .
$$

\section{CONClusion}

In this study, we have obtained three traveling wave solutions of FEW and MFEW equations by using $\left(G^{\prime} / G\right)$-expansion method. Modified Riemann-Liouville derivative is preferred for time-space fractional derivatives. Besides, fractional complex transform that is simple and effective, is implemented to convert FPDE into an ODE. We would also like to say that the method can be used for many other nonlinear fractional differential equations. Another point that is worthy of being emphasized is that the acquired solutions in this work have not been reported in the literature up to now. 
As a final remark that the exactness of the obtained solutions is verified by substituting them back into the original equation. It can be said that they satisfy the FEW and MFEW equations under consideration.

\section{ACKNOWLEDGEMENT}

The author thanks the editor and the referees for the helpful comments and suggestions, which allowed to improve the paper.

\section{REFERENCES}

[1] F. Abdolrazaghi and A. Razani, "On the weak solutions of an overdetermined system of nonlinear fractional integro-differential equations." Miskolc Mathematical Notes, vol. 20, no. 1, pp. 3-16, 2019, doi: 10.18514/MMN.2019.2755.

[2] E. Aksoy, A. C. Çevikel, and A. Bekir, "Soliton solutions of $(2+1)$-dimensional timefractional Zoomeron equation.” Optik, vol. 127, no. 17, pp. 6933-6942, 2016, doi: 10.1016j.ijleo.2016.04.122.

[3] R. L. Bagley and P. J. Torvik, "A theoretical basis for the application of fractional calculus to viscoelasticity." J. Rheol., vol. 27, no. 3, pp. 201-210, 1983, doi: 10.1122/1.549724.

[4] R. Baillie, "Long memory process and fractional integration in econometrics." J. Econom., vol. 73, no. 1, pp. 5-59, 1996, doi: 10.1016/0304-4076(95)01732-1.

[5] E. Barkari, R. Metzler, and J. Klafter, "From continuos time random walks to the fractional Fokker-Planck equation.” Phys. Rev. E, vol. 1, pp. 132-138, 2000, doi: 10.1103/physreve.61.132.

[6] G. W. Bohannan, "Analog fractional order controller in temperature and motor control applications." J. Vib. Control, vol. 14, pp. 1487-1498, 2008, doi: 10.1177/1077546307087435.

[7] R. Gorenflo and F. Mainardi, Essentials of fractional calculus. Preprint to submitted to MaPhySto Center., 2000.

[8] B. Guo, X. Pu, and F. Huang, Fractional partial differential equations and their numerical solutions. Beijing, China: Science Press, 2011.

[9] J.-H. He, S. Elegan, and Z. Li, "Geometrical explanation of the fractional complex transform and derivative chain rule for fractional calculus." Phys. Lett. A, vol. 376, no. 4, pp. 257-259, 2012, doi: 10.1016/j.physleta.2011.11.030.

[10] V. Ho, N. V. Huynh, and P. Le, "Solutions of homogeneous fractional p-Kirchoff equations in RN.” Miskolc Math. Notes, vol. 20, no. 2, pp. 957-968, 2019, doi: 10.18514/MMN.2019.2869.

[11] K. Hosseini and Z. Ayati, "Exact solutions of space-time fractional EW and modified EW equations using Kudryashov method." Nonlinear Sci. Lett. A, , vol. 7, no. 2, pp. 58-66, 2016.

[12] G. Jumarie, "Table of some basic fractional calculus formulae derived from a modified RiemannLiouville derivative for non-differentiable functions.” Appl. Math. Lett., vol. 22, no. 3, pp. 378385, 2009, doi: 10.1016/j.aml.2008.06.003.

[13] V. Kokilashvili, A. Meskhi, and M. Ragusa, "Weighted Extrapolation in Grand Morrey Spaces and Applications to Partial Differential Equations." Rend. Lincei Mat. Appl., vol. 30, no. 1, pp. 67-92, 2019.

[14] A. Korkmaz, "Exact solutions of space-time fractional EW and modified EW equations." Chaos Solitons Fract., vol. 96, pp. 132-138, 2017, doi: 10.1016/j.chaos.2017.01.015.

[15] K. B. Oldham and J. Spanier, The fractional calculus.Theory and Applications of Differentiation and Integration to Arbitrary Order. New York-London: Academic Press, 1974.

[16] Özkan Güner and H. Atık, "Soliton solution of fractional-order nonlinear differential equations based on the exp-function method." Optik, vol. 127, no. 20, pp. 10076-10083, 2016, doi: 10.1016/j.ijleo.2016.07.070. 
[17] I. Podlubny, Fractional differential equations. San Diego: Academic Press, 1999.

[18] M. A. Ragusa, "Necessary and sufficient condition for a VMO function." Applied Math. Comput., vol. 218, no. 24, pp. 11 952-11 958, 2012, doi: 10.1016/j.amc.2012.06.005.

[19] K. Raslan, K. Ali, and M. Shallal, "The modified extended tanh method with the Riccati equation for solving the space-time fractional EW and MEW equations." Chaos Solitons Fract., vol. 103, pp. 404-409, 2017, doi: 10.1016/j.chaos.2017.06.029.

[20] M. Wang, X. Li, and J. Zhang, "The $\left(\frac{G^{\prime}}{G}\right)$-expansion method travelling wave solutions of nonlinear evolution equations in mathematical physics." Phys. Lett. A, vol. 372, no. 4, pp. 417-423, 2007, doi: 10.1016/j.physleta.2007.07.051.

[21] M. Younis, "A new approach for the exact solutions of nonlinear equations of fractional order via modified simple equation method." Appl. Math., vol. 5, pp. 1927-1932, 2014, doi: 10.4236/am.2014.513186.

[22] H. Zhang, "New application of the $\left(G^{\prime} / G\right)$-expansion method." Commun. Nonlinear Sci. Numer. Simulat., vol. 14, pp. 3220-3225, 2009, doi: 10.1063/1.4794947.

[23] S. Zhang and H. Zhang, "Fractional sub-equation method and its applications to nonlinear fractional PDEs.” Phys. Lett. A, vol. 375, pp. 1069-1073, 2011, doi: 10.1016/j.physleta.2011.01.029.

Author's address

Bahar Karaman

E-mail address: bahar_korkmaz@eskisehir.edu.tr

Department of Mathematics, Eskişehir Technical University, Eskişehir 26470, Turkey 